Motivationally-Relevant Domains of Positive Affectivity are Differentially Related to Social Anxiety Symptoms

Corinne N. Carlton ${ }^{1}$, Janey E. Dike ${ }^{1}$, Matthew F. D. Brown ${ }^{2}$, Kasey Stanton ${ }^{1,2}, \&$ John A. Richey $^{1}$

${ }^{1}$ Department of Psychology, Virginia Polytechnic Institute and State University, Blacksburg, Virginia, United States

${ }^{2}$ Department of Psychology, Western University, London, Ontario, Canada

Correspondence concerning this paper should be addressed to Corinne Carlton, Virginia Tech, Department of Psychology, 109 Williams Hall, Blacksburg, VA, 24061. Email: corinnecarlton@vt.edu. 

ANXIETY

\begin{abstract}
Background: This study investigated the extent to which specific facets of positive affectivity (PA) demonstrate differential relationships with social anxiety symptomatology as well as social functioning. Following the conceptual framework of the Broaden and Build theory, as well as prior work demonstrating reward-based linkages to specific PA subdomains, we hypothesized that motivationally-valenced PA facets would show distinct associations with social anxiety and social functioning measures.

Methods: Two samples $(N=446$ and $N=375)$ completed self-report measures of PA, social anxiety, internalizing symptoms, and social functioning. Correlational, multiple and logistic regression, and contrast analyses of correlated correlation coefficients were used to identify the presence and magnitude of relationships between PA facets and symptom measures.

Results: Relationships between social anxiety and specific subdomains of PA appeared to depend on the motivational relevance of each facet. Specifically, self-assurance was associated with social anxiety symptoms above and beyond other PA facets and negative affect. Additionally, contrast analyses indicated that motivationally-valenced PA facets were stronger predictors than non-motivationally-valenced PA facets for social anxiety symptoms.

Conclusions: These results demonstrate a statistically significant divergence between motivationally-valenced subdomains of PA and non-motivationally-valenced subdomains of PA, as they relate to social anxiety symptom severity.
\end{abstract}

Keywords: positive affect; social anxiety; motivation; social functioning 


\section{Motivationally-Relevant Domains of Positive Affectivity are Differentially Related to Social}

\section{Anxiety Symptoms}

Social anxiety disorder (SAD) is characterized by intense fear of one or more social situations (American Psychiatric Association, 2013), and is among the most common anxiety disorders, with approximately $12 \%$ of the population in the United States meeting diagnostic criteria for SAD in their lifetime (Kessler et al., 2005). Prior work has consistently indicated that symptoms of SAD are engaged effectively by CBT, which is currently considered the "gold standard" form of intervention (Gordon, Wong, \& Heimberg, 2014). Although treatment with CBT tends to functionally reduce symptoms of SAD at about a $50 \%$ rate of diagnostic remission (Loerinc et al., 2015), a large proportion of individuals experience persistent symptoms and even relapse within the first year following treatment (Heimberg et al., 1998; Otto et al., 2000). Moreover, recent meta analyses have demonstrated that SAD has one of the lowest remission rates as compared to other anxiety disorders when treated with CBT (Loerinc et al., 2015; Springer, Levy, \& Tolin, 2018). Taken together, the available evidence from RCTs, meta analyses, and related clinical studies indicate that although CBT continues to hold significant promise, current treatment approaches do not comprehensively target mechanisms of SAD genesis and maintenance in affected adults. This gap in efficacy further implies an urgent need to identify and critically evaluate novel therapeutic mechanisms. Perhaps as a consequence, a considerable amount of recent empirical work has evaluated novel mechanisms of change in SAD treatment focusing on deficits on positive affect, which appears to be unique to SAD relative to the other anxiety disorder diagnoses (Brown, Chorpita, \& Barlow, 1998; Kotov et al., 2017; Watson \& Naragon-Gainey, 2010). Inasmuch as most interventions within the CBT-family 
of approaches target negative rather than positive affective dysfunction (Gordon, Wong, \& Heimberg, 2014; Moscovitch, Antony, \& Swinson, 2009), ignoring this distinguishing feature of the diagnosis could potentially relate to the relatively modest rates of remission seen among SAD samples. However, most prior work has evaluated positive affect as a global or unitary construct, and as a result little is known about the constituent components of PA and how they relate to SAD symptomatology despite considerable evidence illustrating that PA is comprised of multiple distinct facets (e.g., Watson \& Clark, 1999; Stanton, Stasik-O’Brien, Ellickson-Larew, \& Watson, 2016). Accordingly, the purpose of this study is to probe the relationship between specific components of PA and social anxiety symptoms as well as social dysfunction, in order to advance understanding of the relationship between deficits in positive emotion and SAD.

In general, affective experience can be broadly defined by anticorrelated, although not totally orthogonal dimensions of positive and negative affect, which can be assessed over a number of different timeframes (e.g., as a current, past day, as a trait; Watson et al., 1999). In the current study, we focus on relatively stable individual differences in the tendency to experience specific types of affect, which is referred to as "affectivity." Positive affectivity (PA) is a broad term used to represent distinct, albeit interrelated positive emotional states such as feelings of joyfulness, excitement, energy and enthusiasm, whereas negative affectivity (NA) refers to feelings of sadness, fear, guilt, and anger (Watson, Clark, \& Tellegen, 1988; Watson \& NaragonGainey, 2010, 2014). Experiences of NA have been widely documented among multiple psychiatric disorders such as depression, anxiety, trauma- and stress-related disorders (Watson, 1988), and are also reported by individuals with other DSM-5 diagnoses even if NA experiences are not included directly within the criteria for those disorders (e.g., attention- 
deficit/hyperactivity disorder; Kotov et al., 2017; Stanton, 2020). As such, NA appears to be a non-specific feature characterizing or broadly associated with multiple psychiatric conditions (Brandes \& Tackett, 2019; Kotov, Gamez, Schmidt, \& Watson, 2010). In contrast, PA appears to show greater specificity in its relationships with certain forms of psychopathology, as low levels of PA appear to be relatively specific to depression, schizophrenia spectrum disorders and SAD (Brown, Chorpita, \& Barlow, 1998; Kotov et al., 2017; Watson \& Naragon-Gainey, 2010). Along these lines, even though all anxiety disorder diagnoses have been associated with elevated levels of NA (Watson, 1988), PA deficits appear to be unique to SAD relative to the remaining DSM-5-defined anxiety disorders (Brown, Chorpita, \& Barlow, 1998). Consistent with this idea, samples of adults with SAD demonstrate a reduced ability to sustain positive emotions and experiences as compared to individuals with other anxiety disorders (Eisner, Johnson, \& Carver, 2009). These associations for PA and social anxiety appear robust even when accounting for comorbid forms of psychopathology such as depression (Kashdan, 2007; Watson \& NaragonGainey, 2010). Moreover, individuals with SAD often report decrements in the enjoyment and motivational pursuit of social interactions, and in overall expression of PA (Trew \& Alden, 2012). Individuals with SAD also demonstrate negative interpretations of positive social events, which have been shown to be mediated by low PA and are implicated in generally worse social relationships (Alden, Taylor, Mellings, \& Laposa, 2007). Further, memory of positive feedback is eroded in individuals with elevated social anxiety (Glazier \& Alden, 2016). This wide constellation of deficits in social functioning may contribute significantly to the degradation of social relationships among affected individuals and may also delay the development of new social relationships (for review see Alden \& Taylor, 2004). 
In addition to concurrent associations between PA and SAD symptomatology, several recent studies further suggest that PA deficits may represent an actionable target of change in interventions, and may thus represent a causal or potentially maintaining factor in the disorder. For example, a study by Taylor and colleagues (2017) demonstrated that individuals who reported a greater propensity to experience positive emotions prior to treatment with CBT displayed larger reductions in anxiety symptoms as compared to individuals reporting lower levels of positive emotions. An additional study indicated that among individuals with SAD, change in positive emotion was the most robust predictor, above and beyond reduction of anxiety symptoms, of increases in connectedness and engagement in future social activities (Taylor, Pearlstein, \& Stein, 2017). Further, recent research by Strege and colleagues (2018) demonstrated that improvements in PA related to significant improvements in SAD symptoms in a small pilot study, highlighting the utility of targeting PA as an actionable mechanism of change in intervention (Strege et al., 2018).

Despite the increasingly accepted linkage between PA and SAD symptomatology (and related social dysfunction), most of the available research examining the interface between SA symptoms and PA has focused solely on broad and global ratings of positive affective experiences, even though prior work has established that positive affective experiences are comprised of distinct facets or sub-domains of this construct (Stanton et al., 2016). This is problematic in that focusing solely on global indices of PA may preclude identification of specific actionable targets within the broader PA construct that may eventually lead to improvements in SAD symptoms when targeted directly. When considering the breakdown of the PA-SAD relationship into distinct facets, the Broaden and Build theory (Fredrickson, 1998, 
2001, 2013) provides a precedent for identifying particular aspects of PA that may be specifically related to social anxiety symptomatology. Specifically, the Broaden and Build theory posits that certain specific positive emotions (e.g., joy, love, interest, contentment, pride, serenity) share a particular capacity to broaden momentary thought-action repertoires (i.e., the ability to reflect and choose an appropriate response in the moment), and thus facilitate pursuit of and behavioral exposure to novel enjoyable activities. The process of increasing the associative strength between a particular appetitive thought and the enjoyable experience of its resultant action is theorized to broaden experiences by driving exposure to manifold enjoyable activities. In turn, the exposure to pleasurable experience is then theorized to build or culminate in enduring personal resources (e.g., social skills, cognitive capacities). For example, a positive emotion such as curiosity may lead to increases in time spent reading across various content areas (i.e. broadening of experience), which leads to increased substantive knowledge in those domains (i.e. building enduring personal resources). More specific to the social domain, positive emotions such as joy may initiate an interest in pursuing novel social actions and relationships (broadening), which then leads to enhancements in social skills, support networks and social knowledge (building).

In light of these factors, appetitive motivational states within the broader PA construct such as enthusiasm or interest may hold particular relevance, to the extent that they are required for the creation of the thought-action connections that are central to Broaden and Build theory. For example, the feeling of joy may specifically produce motivation for exploration and playfulness, which in turn leads to heightened creativity and the development of new social bonds (Fredrickson, 2004). Based upon the central tenets of Broaden and Build theory, as well as 
prior work illustrating that motivational quality of social interaction is diminished in $\mathrm{SAD}$, one would therefore predict that social anxiety symptomatology and social functioning would be inversely related to PA domains that are specifically linked to appetitive motivational states (e.g., self-assurance, joy), whereas sub-domains of PA (e.g., attentiveness) that are less related to reward-driven or appetitive motivation would not. Supporting the notion of reward-relevant PA states having an impact on social anxiety symptoms and social functioning, previous work has indicated that specific activated PA facets (e.g., self-assurance) are linked to reward seeking and to the dopaminergic system, which governs motivational and approach-related behaviors (see DeYoung, 2013). This is of particular relevance here, in that prior work has established that reward neurobiology and its behavioral correlates are functionally suppressed in SAD (Cremers, Veer, Spinhoven, Rombouts, \& Roelofs, 2015; Richey et al., 2017) and dysfunction in reward circuitry has been linked to motivational deficits exhibited in SAD (for review see Carlton, Sullivan-Toole, Ghane, \& Richey, 2020). Together, predictions posited by the Broaden and Build theory, combined with results from previous work demonstrating the relationship between blunted reward functioning and social anxiety, suggest that there may be a distinct linkage between motivational states contained within the PA construct and social anxiety symptoms.

In sum, considerable evidence now suggests that SAD bears a unique association with low PA and that the consequences of this phenomenon may include multiple types of social dysfunction including depletion of social resources (Brown, Chorpita, \& Barlow, 1998; Glazier \& Alden, 2016; Trew \& Alden, 2012). Additionally, it has been demonstrated that improvements in PA specifically impact treatment outcomes for SAD (Strege et al., 2018; Taylor et al., 2017; Taylor, Pearlstein, \& Stein, 2017). Yet, despite the increasing appreciation of the importance of 
the PA-SAD interface, little is known about the specific facets of PA and how they relate to SAD symptomatology. Accordingly, the current study tested the overall hypothesis that, consistent with Broaden and Build theory, distinctive facets of PA that describe appetitive motivational states will demonstrate distinct relationships with social anxiety symptomatology and social dysfunction as compared to PA subconstructs such as attentiveness and serenity that are not characteristic of appetitive or activated motivation. In addition, we aimed to compare the extent to which positive affect facets show specificity in their relations with social anxiety symptoms versus general internalizing (i.e., dysphoria) symptoms. We specifically predicted that appetitive PA facets (as measured by the PANAS-X; Watson and Clark, 1999) would show negative associations with continuous ratings of social functioning, social anxiety, and dysphoric mood. We were mainly interested in determining the extent to which certain PA facets showed differential, and significantly stronger negative associations with these variables than other PA facets. Based on prior work suggesting that joyfulness and liveliness (as indexed by the 'joviality' factor on the PANAS-X) have been most positively linked to manic symptoms and negatively correlated with depressive symptoms (Stanton et al., 2016; Watson et al., 2010), we also predicted that the facet of joviality would show strong negative relations to social anxiety and dysphoric symptoms, as well as with social functioning levels. In light of the Fredrickson Broaden and Build theory of positive emotions (1998, 2001, 2013), and on work illustrating diminished social reward seeking in SAD (Richey et al., 2017), as well as research indicating that the self-assurance domain of PA is related to the dopaminergic system and reward seeking in general (DeYoung, 2013; Stanton and Watson, 2015; Stanton et al., 2016), we specifically hypothesized that the self-assurance facet—which is defined by elements of confidence and boldness - would show a robust negative relationship to symptoms of social anxiety and 
dysphoria, as well as measures of social functioning. In comparison, given that serenity (i.e., feelings of calm, relaxation, ease) tends to function more as a facilitative context for consummatory and affiliative needs to be met (Depue \& Morrone-Strupinsky, 2005) and that attentiveness (i.e., alertness; conscientiousness) has demonstrated less specificity, and related to broader internalizing disorders (Stanton et al., 2016), we expected that the attentiveness and serenity facets would be less strongly linked to these measures, even though they also are PA states overlapping with joviality and self-assurance. We also examined associations for PA facets with clinically elevated levels of social anxiety and dysphoric symptoms, and consistent with our analyses using dimensional symptom ratings, we again predicted that self-assurance scores would uniquely predict a decreased likelihood of membership in the "clinically" elevated social anxiety or dysphoric group above and beyond the other PA facets. Moreover, we predicted that the motivationally-valenced facets of self-assurance and joviality would serve as the strongest predictors of social anxiety and social functioning as compared to other facets.

\section{Method}

\section{Participants}

Participants included respondents from two samples: An undergraduate sample $(N=447)$ that was recruited through the psychology department pool at a Canadian university, and a community sample $(N=375)$ that was recruited using Amazon Mechanical Turk. Specific details pertaining to each sample are described below.

Undergraduate sample. This sample consisted of 447 undergraduates, with an average participant age of 18.6 years $(S D$ 1.6). Within this sample, the majority of participants identified as female $(n=303 ; 67.8 \%)$. Data regarding racial and ethnic identity were gathered in a manner 
consistent with Statistics Canada's recommendations for the regional location of this university, as advised by this institution's IRB. The racial and ethnic identity breakdown of this sample is as follows: White or of European descent (40.9\%), East Asian (e.g., Chinese descent; 28.2\%), South Asian (e.g., Indian descent; 16.6\%), and Arabic/Middle Eastern (4.5\%). The remaining $9.8 \%$ percentage of participants identified as a range of other ethnicities. Regarding therapeutic or psychopharmacological treatment for mental health issues, $7.6 \%$ of participants in this sample indicated that they were currently receiving psychotherapy services, while $6.7 \%$ of participants reported that they were currently being prescribed medication at the time of data collection.

Community sample. The second sample consisted of 375 community adults. The average participant age was 39.1 years $(S D=12.1)$, with $53.6 \%$ of the sample identified as male $(N=201)$. The majority of participants identified as White or European American $(73.3 \%)$, $10.1 \%$ identified as Black or African-American, $8.0 \%$ as Asian/Asian-American, with the remaining percentage of the sample endorsing other identities. Additionally, $5.1 \%$ identified as Hispanic or Latinx. Regarding highest level of education obtained, $41.6 \%$ of the sample reported having a bachelor's degree; 32.3\% reported having a vocational degree, an associate degree, or some college; $15.2 \%$ reported having a high school diploma or less education, and $10.9 \%$ reported having a master's degree or higher. Additionally, 77.3\% of participants reported fulltime employment, $11.5 \%$ reported part-time employment, and $11.2 \%$ reported being unemployed. Regarding mental health treatment status, approximately $13.1 \%$ of participants endorsed that they were currently receiving mediation to treat psychiatric issues at the time of this study. Furthermore, $7.7 \%$ of participants reported that they were currently receiving some form of psychotherapy during this period of data collection. 


\section{Assessment of Positive and Negative Affectivity}

The PANAS-X (Watson and Clark 1999) was used to measure NA and facets of PA in both samples. The specific PANAS PA scales include Joviality (8 items; e.g., joyful, delighted), Self-Assurance (6 items; e.g., proud, confident), Attentiveness (4 items; e.g., concentrating, alert), and Serenity (3 items; e.g., calm). Participants were also asked to complete some items related to NA. However, they were only asked to respond to items used to score the PANAS-X general NA scale (10 items; e.g., irritable, ashamed). Participants indicated the extent to which they have experienced each affectivity term "in general" on a 5-point Likert-type scale ranging from 1 (very slightly or not at all) to 5 (extremely). Therefore, these ratings reflect relatively stable individual differences in tendencies to experience positive and negative mood states as noted. Additionally, scales of the PANAS-X have been demonstrated to have excellent internal consistency ( $a=$ ranging from .83 to .90 ; Watson \& Clark, 1999). Coefficient alpha estimates for all PANAS-X scales exceeded .70 across both samples (Negative Affectivity, undergraduate sample $a=.84$, community sample $a=.92$; Joviality, undergraduate sample $a=.92$, community sample $a=.95 ;$ Self-Assurance, undergraduate sample $a=.84$, community sample $a=.87$; Attentiveness, undergraduate sample $a=.74$, community sample $a=.80$; and Serenity, undergraduate sample $a=.83$, community sample $a=.90$ ).

\section{Symptom Assessment}

Participants from both samples completed select scales from the Expanded Version of the Inventory of Depression and Anxiety Symptoms (IDAS-II; Watson et al., 2007; Watson et al., 2012), a widely used and validated measure assessing a range of depressive, anxious, obsessivecompulsive and other symptom dimensions (Nelson, O’Hara, \& Watson, 2018). Scales from the IDAS-II that were administered and incorporated into our analyses here included the Dysphoria 
(10 items; e.g., "felt depressed"; "blamed myself for things") and Social Anxiety (5 items; e.g. "felt self-conscious knowing others were watching me") scales, both of which were expected to show robust negative associations with PA and social functioning. In our analyses, we examine associations for PA facets both with (a) dimensional scores obtained from each IDAS-II scales (i.e., sum scores using all items for each scale) as well as (b) categorical ratings of Social Anxiety and Dysphoria based on clinical cutoffs indicating significant levels of each respective symptom type (Stasik et al., 2019). Note that the IDAS-II includes six items that can be used to score Social Anxiety; however, we used the 5-item version of the Social Anxiety scale from the original IDAS because clinical cutoff scores (i.e., a score of 18.5 or higher) have been identified only for this scale version (Stasik et al., 2019). Also note that elevated scores on Dysphoria (i.e., cutoff score of 28.5 or higher) do not map onto a single DSM-based diagnostic cutoff, but rather, reflect elevated levels of distress-based symptoms listed as criteria for many different DSM disorders (e.g., depressive disorders, generalized anxiety, posttraumatic stress disorder).

Participants were asked to indicate how much they experienced each symptom over the past 2 weeks using a 5-point scale ranging from 1 (not at all) to 5 (extremely). The IDAS-II scales also have demonstrated strong internal consistency ( $a$ = ranging from .80 to .90; Watson et al., 2012). In this study the IDAS-II also showed excellent internal consistency across both samples (Social anxiety, undergraduate sample $a=.88$, community sample $a=.90$; Dysphoria, undergraduate sample $a=.91$, community sample $a=.91)$.

\section{Social Functioning Assessment}

Participants from the undergraduate sample also completed several other measures of social functioning. This included the Social Functioning Questionnaire (SFQ; Tyrer et al., 2005), a widely used measure of functioning. When completing the SFQ, participants are asked to 
answer eight items about their social functioning in the past 2 weeks (e.g., "I have difficulties in getting and keeping close relationships") using a 4-point Likert-type scale ranging from 1 (most of the time) to 4 (not at all). The internal consistency of the SFQ in this study was shown to be acceptable $(a=.66)$. Additionally, participants from the undergraduate samples completed the Dysfunctional Attitude Scale (DAS-17; de Graaf, Roelofs, \& Huibers, 2009) to assess levels of perfectionism and dependency, both of which are related to poorer social functioning. Participants were asked to answer 17 items related to their attitudes about different aspects of life on a 7-point Likert-type scale ranging from 1 (fully disagree) to 7 (fully agree). The DAS-17 has demonstrated excellent internal consistency across other studies (de Graaf, Roelofs, \& Huibers, 2009). Coefficient alpha estimates for the DAS-17 Perfectionism and Dependency subscales were .90 and .84 , respectively.

\section{Results}

\section{Overview of Analytic Strategy}

First, Pearson correlations between measures of PA facets and general NA were computed in both samples to determine the degree to which scores on each of these measures were interrelated. Second, we computed correlations for the PA facet measures with measures of social anxiety, dysphoria, and social functioning. Correlations for PA measures with other continuously scored variables are Pearson correlations, whereas point biserial correlations were computed to examine associations with dichotomously scored variables (i.e., clinical levels of social anxiety and dysphoria). Standardized beta weights are also reported from multiple regression analyses to characterize the extent to which PA facets are associated with internalizing symptom scores (i.e., social anxiety and dysphoria) as well as scores on measures 
of self-reported social functioning. Additionally, we report odds ratios from logistic regression analyses with dichotomous "clinical" versus "non-clinical" groups for both social anxiety and dysphoria within both samples. Across these analyses, all four PA facet scales (i.e., Joviality, Self-Assurance, Attentiveness, and Serenity), as well as the broad NA scale were entered concurrently as predictors in all regression analyses. This approach identified the unique, incremental predictive power of each PA scale when assessing the association with internalizing symptoms and social functioning. By including general NA scores in these analyses, we also were able to examine the extent to which PA measures showed incremental predictive power beyond negative affectivity.

In the logistic regression analyses, an odds ratio of less than 1.00 indicates that higher scores on a PA facet were negatively associated with being grouped in the "clinical" range for social anxiety or dysphoria. Conversely, an odds ratio greater than 1.00 indicated that higher PA facet scores were associated with an increased chance of being grouped into the "clinical" range for social anxiety or dysphoria. Of note, of the 447 undergraduate participants, 67 participants had scores within the clinical range for social anxiety, and 195 fell within the clinical range for dysphoria; 380 fell within the nonclinical range for social anxiety, and 252 fell within the nonclinical range for dysphoria. Additionally, of the 375 participants in the community sample 20 participants had elevated social anxiety scores on the IDAS-II within the clinical range, and 64 fell within the clinical range for dysphoria; 355 fell within the nonclinical range for social anxiety, and 311 fell within the non-clinical range for dysphoria. Despite this, these rates are generally consistent with previous studies (e.g., Gren-Landell, Aho, Andersson, \& Svedin, 2011). Additionally, all scores on the PA facet scales, as well as on the general NA scale were standardized by subtracting the mean prior to conducting the logistic regression, in order to 
ensure that all scales were on a common metric. Lastly, contrast analyses were run to evaluate the significance of the difference between correlated correlation coefficients using the Meng, Rosenthal, and Rubin (1992) approach, which identified the extent to which specific PA factors (motivationally relevant versus non-motivationally relevant) were more strongly correlated with the dependent measures of interest here, despite their mutual correlation by virtue of their mutual association as facets of PA.

\section{Correlations Among the Positive and Negative Affectivity Scales}

Table 1 indicates that all PA facets were strongly inter-correlated ( $r s$ ranged from .38 to .78). In particular, measures of high arousal forms of PA such as Joviality and Self-Assurance correlated very strongly in both samples. All PA facet scores also correlated negatively with PANAS-X NA in both the undergraduate and community samples, although the magnitude of these associations varied widely (e.g. $r s$ ranged from -.56 to -.11), with PANAS-X Serenity correlating most strongly with NA of any of the PA facets $(r=-.29$ and -.56 in the undergraduate and community datasets, respectively).

*****Insert Table 1 About Here ***** $^{*}$

\section{Correlations for the PA Facets with Internalizing Symptoms and Self-Reported Social Functioning}

Correlational analyses examining associations for PA with symptoms and social functioning are provided in Table 2. These analyses indicated that all of the PA scales generally 
demonstrated negative correlations with both self-reported measures of internalizing psychopathology and social functioning measures. Additionally, strong positive correlations (i.e., $>$.30) were observed in both samples between negative affectivity and self-reported measures of internalizing psychopathology and social functioning measures.

*****Insert Table 2 About Here*****

\section{Regression Results Predicting Internalizing Symptoms and Social Functioning from the PA Facets and Negative Affectivity}

Results from regression analyses are displayed in Table 3 (standardized beta weights from multiple regressions) and Table 4 (odds ratios from logistic regressions). The following results, presented in distinct sections for social anxiety, dysphoria, and social functioning, provide specific descriptions of the pattern of associations for PA with each dependent measure.

*****Insert Tables 3 \& 4 About Here A**** $^{*}$

Social anxiety. Multiple regression results regarding social anxiety indicate that in both the undergraduate and community samples, PANAS-X Self-Assurance scores appeared to be the most strongly associated with social anxiety symptoms $(\beta=-.20, p<.001$ and $-.15, p<.05$, respectively). Moreover, results from the logistic regression analyses further support the 
specificity of the Self-Assurance PA facet as strongly associated to social anxiety (odds ratio of .59 and .29 in the undergraduate and community samples, respectively; $p<.01$ and $p<.05$, respectively), such that higher scores on the Self-Assurance facet were negatively associated with being in the "clinical" range for social anxiety. Of note, only in the undergraduate sample did the Attentiveness PA facet emerge as significantly associated to social anxiety through logistic regression analysis (odds ratio 1.56; $p<.05$ ); this can be interpreted such that higher scores on the Attentiveness PA facet reflect a positive association for falling in the "clinical" range for social anxiety. It should also be noted that in the multiple regression results, higher levels of negative affectivity were also associated with social anxiety symptoms $(\beta=.49$ and .60 in the undergraduate and community samples, respectively; both at $p<.001$ ). Additionally, the logistic regression results further support this idea, such that higher NA scores are associated with an increased likelihood of being in the "clinical" range for social anxiety (odds ratio of 2.98 and 2.62 in the undergraduate and community samples, respectively; $p<.001$ ). With the exception of the attentiveness PA facet results described above, analyses were consistent across samples in regard to social anxiety.

Dysphoria. In Table 3, multiple regression analyses indicate some differences in the patterns of results across samples. For example, results regarding Joviality, Attentiveness, and Serenity were not uniform across samples such that results from the community sample indicated that the Joviality and Attentiveness PA facets predicted dysphoria symptoms to some degree (Joviality: $\beta=-.15, p<.05$; Attentiveness: $\beta=-.09, p<.05$ ), whereas this was not the case in the undergraduate sample. Additionally, results from the undergraduate sample indicated that Serenity was the only significant predictor of social anxiety at a $p<.05$ level $(\beta=-.10)$, which were not consistent with the community sample. Thus, results were somewhat inconsistent across 
samples and the observed coefficients were relatively weak in magnitude. Moreover, logistic regression results (Table 4) did not indicate that any PA facets significantly predicted group membership of "clinical" levels of dysphoria in either sample. NA was associated positively with dysphoria in both samples and in both multiple and logistic regression analyses $(\beta=.60$ and .70 in the undergraduate and community samples, respectively; both $p<.001$; odds ratio of 4.49 and 6.94 in the undergraduate and community samples, respectively; both $p<.001$ ).

Social functioning. As noted earlier, social functioning measures were only administered in the undergraduate sample. Therefore the following results pertain to the multiple regression analyses conducted in that sample only. In addition to the SFQ, participants from the undergraduate samples completed the DAS-17 in order to assess for levels of perfectionism and dependency, which have both been associated with poorer social functioning (de Graaf, Roelofs, $\&$ Huibers, 2009). For SFQ scores, the PA facets of Joviality $(\beta=-.19)$, Attentiveness $(\beta=-.20)$, and Serenity $(\beta=-.17)$ all emerged as significant predictors of social functioning at a $p<.001$ level. NA also emerged as a significant predictor $(\beta=.44)$ at $p<.001$, such that higher selfreported ratings of NA significantly predicted higher scores (i.e., poorer social functioning) on the SFQ.

Regarding the DAS-17 Perfectionism scale, only PANAS-X Joviality emerged as a significant PA facet predictor of scores on this measure $(\beta=-.17 ; p<.01)$, such that higher selfreported scores on the Joviality PA facet predicted lower scores (i.e., less perfectionist tendencies) on the DAS-17 Perfectionism scale. Additionally, NA also emerged as a significant positive predictor of DAS-17 Perfectionism $(\beta=.36, p<.001)$. Finally, on the DAS-17 Dependency scale, only Serenity emerged as a significant PA facet predictor of dependency $(\beta=$ $-.14, p<.01)$. This can be interpreted to mean that higher scores on the Serenity PA facet predict 
lower scores (i.e., less dependence) on the DAS-17 Dependency scale. As with other measures of social functioning, described above, PANAS-X NA also emerged as a strong predictor of dependency $(\beta=.28, p<.001)$, meaning that higher self-reported NA significantly predicted higher scores (i.e., more dependence) on the DAS-17 Dependency scale.

\section{Contrast Analyses}

Using Meng, Rosenthal, and Rubin's (1992) method of comparing non-independent correlations, we examined if correlations resulting from motivationally-valenced PA facets (i.e., self-assurance and joviality) and dependent measures of interest were significantly stronger than correlations of non-motivationally-valenced PA facets (i.e., attentiveness and serenity). Results are presented in Table 5. Through this approach, correlations between motivationally-valenced PA facets and both dimensional and dichotomous social anxiety symptoms emerged as significantly stronger than the correlations of non-motivationally-valenced PA facets with these dependent measures in the undergraduate sample (dimensional social anxiety: $\mathrm{z}=-1.90, p \leq .05$; dichotomous social anxiety: $\mathrm{z}=-2.37, p \leq .01)$. However, this relationship also emerged for the community sample for dimensional social anxiety symptoms $(\mathrm{z}=1.70, p \leq .05)$, but not for dichotomous ([i.e., clinical]; $\mathrm{z}=-0.44, p=.33$ ) social anxiety. Additionally, this relationship is also found in the community sample regarding dimensional dysphoria and in the undergraduate sample regarding dichotomous dysphoria (dimensional dysphoria in community sample: $\mathrm{z}=$ $2.51, p \leq .01$; dimensional dysphoria in undergraduate sample: $\mathrm{z}=0.58, p=.28$; dichotomous dysphoria in community sample: $\mathrm{z}=0.30, p=.39$; dichotomous dysphoria in undergraduate sample: $\mathrm{z}=1.75, p \leq .05)$. These contrast analyses indicated that there was a significantly stronger relationship between motivationally-valenced PA facets and measures of social anxiety within the undergraduate sample; however, this pattern was not the same within the community 
sample. Although motivationally-valenced PA facets were more strongly related to dimensional symptoms of social anxiety than non-motivationally-valenced facets within the community sample, these facets show a divergence from the undergraduate sample when looking at clinical levels, such that they are more strongly associated with clinical levels of dysphoria rather than clinical levels of social anxiety.

\section{*****Insert Table 5 About Here ${ }^{* * * * *}$}

\section{Discussion}

The primary focus of this study centered on the specificity of PA facets in their associations with social anxiety and social functioning measures. While at the broadest level, we expected that all PA facets would be negatively related to social anxiety, dysphoria and social functioning, we more specifically expected that subdomains of PA related to appetitive motivational states would demonstrate significantly greater levels of association to SA symptomatology and social dysfunction. Results of this study supported this broad initial hypothesis, as all PA facets demonstrated a significant negative correlation across all measures of social anxiety, internalizing symptoms and social functioning measures. More importantly, results from this study identified several fine-grained associations between subdomains of PA and clinically relevant dependent measures of interest. For example, results indicated that the subdomain of self-assurance, above and beyond other PA facets and NA, was more linked to both dimensional and categorical ratings of SAD. Specifically, lower levels of self-assurance 
emerged as a specific correlate of social anxiety symptoms, and as indicative of an enhanced likelihood of belonging to a clinically elevated group of socially anxious individuals; whereas other PA facets demonstrated weaker or nonsignificant relations with internalizing symptoms and social functioning measures once accounting for overlap amongst facets and with NA in regression analyses. Further, contrast analyses indicated that motivationally-valenced PA facets (including self-assurance) were stronger predictors than other PA facets at predicting social anxiety symptoms. Therefore, our results regarding self-assurance further support both predictions posited by the Broaden and Build theory, as well as previous work investigating reward-based systems and motivational deficits in SAD (e.g., Richey et al., 2019), suggesting that deficits in self-assurance may be linked to heightened rates of social anxiety via rewardbased mechanisms that may also be linked to social deficits inhibiting the development of strong social relationships.

By contrast, differential and non-specific patterns emerged for social functioning measures such that the PA facets of Joviality, Attentiveness, and Serenity were significantly associated with various measures of social functioning. Results regarding joviality were mixed when considering predictions related to the Broaden and Build theory. Specifically, feelings of joviality were inversely linked to social functioning measures, perhaps supporting the work in Fredrickson's (2004) study discussing joy as a motivational agent for the development of new social bonds. However, joviality was only weakly correlated with social anxiety symptoms and was not indicative of clinical group membership for social anxiety. In fact, joviality was more linked to general internalizing symptomology (i.e., dysphoria) than specific socially-valenced anxiety in the community sample. This pattern of results has been seen in other studies examining joviality, wherein feelings of joviality have been shown to be linked more strongly to 
major depressive disorder and generalized anxiety disorder rather than social anxiety disorder (Stanton et al., 2016; Watson, Clark, \& Stasik, 2011). Results regarding the serenity facet were supportive of our predictions. Specifically, we predicted that serenity would not show a strong association with social anxiety nor social functioning measures, nor would it be more strongly correlated when investigated with contrast analyses in predicting social anxiety symptoms, due to previous work suggesting that serenity did not act as a motivational agent (Depue \& MorroneStrupinsky, 2005). Results from this study indicate that serenity was weakly associated with only social functioning measures, but not social anxiety symptoms. Results regarding attentiveness largely aligned with our predictions and results of previous studies, suggesting that feelings of attentiveness are more generally linked to broad internalizing disorders (Stanton et al., 2016). Contrast analyses revealed an unexpected pattern across samples, such that the younger sample (i.e., undergraduate sample) had significantly stronger relationship between motivationally-valenced PA facets and both dimensional social anxiety symptoms and clinical levels of social anxiety; whereas this same pattern of results was not observed in the community sample. In fact, contrast analyses in the community sample indicated that although motivationally-valenced PA facets were more strongly related to dimensional symptoms, these facets were more strongly related to clinical levels of dysphoria rather than clinical levels of social anxiety. These differential results may be in part due to the trajectory of SAD, which emerges during adolescence (Ollendick \& Hirshfield-Becker, 2002) when sensitivity to social rewards are heightened (Somerville, 2013; Richey et al., 2019); suggesting that perhaps targeting motivationally-valenced PA facets in future interventions may be more profitable in younger populations. 
Critically, findings from this study provide evidence for the specificity of relationships between PA facets that are theorized to induce reward-based behaviors with symptoms of social anxiety. Moreover, and perhaps more importantly, these findings indicate a divergence in the specific associations between PA facets that do not map on as motivational agents and sociallyvalenced anxiety and functioning measures. Specifically, the PA facet that has been shown to have a distinct linkage to the dopaminergic system and reward seeking (i.e., self-assurance) had robust associations with social anxiety symptomology as well as clinical levels of social anxiety symptomology, whereas PA facets that have not been linked to reward or theorized to act as motivational agents tended to have broad associations with social functioning measures. This divergence further suggests specificity regarding potential therapeutic targets between the domains of anxiety and functional impairment. Future research should examine this distinction in the context of therapeutic intervention enhancement. Furthermore, given the relative success of improved understanding of hedonic deficits in other disorders such as depression has advanced understanding of treatment targets, results presented here may bear upon future treatment development efforts in the realm of social anxiety (see Stanton et al., 2016; Gruber, 2011; Carl, Soskin, Kerns, \& Barlow, 2013). Indeed, in addition to previous work suggesting that improvements in PA produce positive treatment outcomes for individuals with $\mathrm{SAD}$, the results presented here further advance this notion by providing anchorable PA targets that are less broad than general PA. Specifically, results of the current study may potentially suggest that targeting improvements in motivationally-valenced aspects of PA may hold particular utility for further understanding SAD.

Overall, results of this study indicated across two relatively large, non-overlapping samples that some facets of PA show comparatively more predictive power than others when 
predicting social anxiety symptoms and social functioning. However, as with any study, results presented here should be evaluated in light of study limitations. For example, although we included a variety of measures of social anxiety, internalizing symptoms, and social functioning that have validated clinical cutoffs, we did not have clinical interview measures to confirm a diagnosis of SAD nor the presence of other disorders. Further, we did not assess some types of PA (e.g., gratitude and warmth) that have been studied widely in recent research (Fredrickson, 2001); therefore, it would be worth examining the degree to which these types of PA show divergent relations from the types of PA considered here. An additional limitation of this study is that it occurred at a single time point. As such, future research would benefit from a longitudinal consideration of these factors in order to clarify the relationship between SAD and PA. Additionally, although this data demonstrates evidence for specificity for the relationship between SAD and PA, causality cannot be inferred without further research. Finally, we demonstrated that the PA facets generally showed significant, weak to moderate negative correlations with symptom and functioning measures. However, due the PA facets being strongly intercorrelated, they were generally weak predictors of most variables in the different sets of regression analyses given that there was relatively little unique variance for each PA facet after accounting for their overlap (see Sleep, Lynam, Hyatt, \& Miller, 2017 for discussion of these issues in other literatures). Given this, we deemed it important to focus on results that were replicable across samples and analyses (e.g., self-assurance negatively predicting social anxiety across samples) so that sample specific findings were not overemphasis when discussing and interpreting key results. For example, PANAS-X Attentiveness significantly predicted social anxiety, but in the logistic regressions only and only in the undergraduate sample. Therefore, it is possible that this finding observed in a single type analysis may not be particularly robust. Thus, 
future research should examine the replicability of the results observed here in other sample types (e.g., clinical samples, adolescent samples) in addition to incorporating longitudinal study design approaches as discussed.

In conclusion, recent studies have demonstrated that improvements in broad PA relate to significant improvements in SAD symptoms (Strege et al., 2018). However, few studies have examined more specific positive affective experiences in the context of SAD (Stanton et al., 2016). In light of this gap, this study aimed to examine the relationship that different types of PA have with social anxiety and social functioning measures in order to more specifically inform targetable mechanisms for research, assessment, and intervention approaches for SAD. Findings from this study highlight the importance of further understanding the influence of motivationally-valenced PA dysfunction in disorders characterized by social deficits such as SAD. These data provide evidence for the specificity of appetitive motivational PA facets in their associations with symptoms of social anxiety. More specifically, these data indicate that self-assurance, in particular, shows distinctive relational and predictive power with social anxiety measures that is stronger than other PA facets and NA. These findings provide scaffolding for future studies to further explicate the relationship of PA with disorders such as SAD, in order to better inform the research, assessment, and treatment protocols targeting these systems. 
ANXIETY

\section{References}

Alden, L. E., \& Taylor, C. T. (2004). Interpersonal processes in social phobia. Clinical Psychology Review, 24(7), 857-882.

Alden, L. E., Taylor, C. T., Mellings, T. M., \& Laposa, J. M. (2008). Social anxiety and the interpretation of positive social events. Journal of Anxiety Disorders, 22(4), 577-590.

American Psychiatric Association, \& American Psychiatric Association. (2013). Diagnostic and statistical manual of mental disorders: DSM-5.

Brandes, C. M., \& Tackett, J. L. (2019). Contextualizing neuroticism in the Hierarchical Taxonomy of Psychopathology. Journal of Research in Personality, 81, 238-245.

Brown, T. A., Chorpita, B. F., \& Barlow, D. H. (1998). Structural relationships among dimensions of the DSM-IV anxiety and mood disorders and dimensions of negative affect, positive affect, and autonomic arousal. Journal of Abnormal Psychology, 107(2), 179.

Carl, J. R., Soskin, D. P., Kerns, C., \& Barlow, D. H. (2013). Positive emotion regulation in emotional disorders: A theoretical review. Clinical Psychology Review, 33(3), 343-360.

Carlton, C. N., Sullivan-Toole, H., Ghane, M., \& Richey, J. A. (2020). Reward Circuitry and Motivational Deficits in Social Anxiety Disorder: What Can Be Learned From Mouse Models?. Frontiers in Neuroscience, 14.

Cremers, H. R., Veer, I. M., Spinhoven, P., Rombouts, S. A., \& Roelofs, K. (2015). Neural sensitivity to social reward and punishment anticipation in social anxiety disorder. Frontiers in Behavioral Neuroscience, 8, 439. 
De Graaf, L. E., Roelofs, J., \& Huibers, M. J. (2009). Measuring dysfunctional attitudes in the general population: The Dysfunctional Attitude Scale (form A) Revised. Cognitive Therapy and Research, 33(4), 345.

Depue, R. A., \& Morrone-Strupinsky, J. V. (2005). A neurobehavioral model of affiliative bonding: Implications for conceptualizing a human trait of affiliation. Behavioral and Brain Sciences, 28, 313-395.

DeYoung, C. G. (2013). The neuromodulator of exploration: A unifying theory of the role of dopamine in personality. Frontiers in Human Neuroscience, 7, 762.

Eisner, L. R., Johnson, S. L., \& Carver, C. S. (2009). Positive affect regulation in anxiety disorders. Journal of Anxiety Disorders, 23(5), 645-649.

Fredrickson, B. L. (1998). What good are positive emotions?. Review of General Psychology, 2(3), 300-319.

Fredrickson, B. L. (2001). The role of positive emotions in positive psychology: The broadenand-build theory of positive emotions. American Psychologist, 56(3), 218.

Fredrickson, B. L. (2004). The broaden-and-build theory of positive emotions. Philosophical Transactions of the Royal Society of London. Series B: Biological Sciences, 359(1449), $1367-1377$.

Fredrickson, B. L. (2013). Positive emotions broaden and build. In Advances in Experimental Social Psychology (Vol. 47, pp. 1-53). Academic Press.

Glazier, B. L., \& Alden, L. E. (2017). Social anxiety and biased recall of positive information: It's not the content, it's the valence. Behavior Therapy, 48(4), 533-543. 
Gordon, D., Wong, J., \& Heimberg, R. G. (2014). Cognitive- Behavioral Therapy for Social Anxiety Disorder: The State of the Science. The Wiley Blackwell Handbook of Social Anxiety Disorder, 475-497.

Gren-Landell, M., Aho, N., Andersson, G., \& Svedin, C. G. (2011). Social anxiety disorder and victimization in a community sample of adolescents. Journal of Adolescence, 34(3), 569577.

Gruber, J. (2011). A review and synthesis of positive emotion and reward disturbance in bipolar disorder. Clinical Psychology \& Psychotherapy, 18(5), 356-365.

Heimberg, R. G., Liebowitz, M. R., Hope, D. A., Schneier, F. R., Holt, C. S., Welkowitz, L. A., ... \& Fallon, B. (1998). Cognitive behavioral group therapy vs phenelzine therapy for social phobia: 12-week outcome. Archives of General Psychiatry, 55(12), 1133-1141.

Kashdan, T. B. (2007). Social anxiety spectrum and diminished positive experiences: Theoretical synthesis and meta-analysis. Clinical Psychology Review, 27(3), 348-365.

Kessler, R. C., Berglund, P., Demler, O., Jin, R., Merikangas, K. R., \& Walters, E. E. (2005). Lifetime prevalence and age-of-onset distributions of DSM-IV disorders in the National Comorbidity Survey Replication. Archives of General Psychiatry, 62(6), 593-602.

Kotov, R., Gamez, W., Schmidt, F., \& Watson, D. (2010). Linking “big” personality traits to anxiety, depressive, and substance use disorders: a meta-analysis. Psychological Bulletin, 136(5), 768.

Kotov, R., Krueger, R. F., Watson, D., Achenbach, T. M., Althoff, R. R., Bagby, R. M., ... \& Eaton, N. R. (2017). The Hierarchical Taxonomy of Psychopathology (HiTOP): a dimensional alternative to traditional nosologies. Journal of Abnormal Psychology, $126(4), 454$. 
Loerinc, A. G., Meuret, A. E., Twohig, M. P., Rosenfield, D., Bluett, E. J., \& Craske, M. G. (2015). Response rates for CBT for anxiety disorders: Need for standardized criteria. Clinical Psychology Review, 42, 72-82.

Meng, X. L., Rosenthal, R., \& Rubin, D. B. (1992). Comparing correlated correlation coefficients. Psychological Bulletin, 111(1), 172.

Moscovitch, D. A., Antony, M. M., \& Swinson, R. P. (2009). Exposure-based treatments for anxiety disorders: Theory and process. Oxford Handbook of Anxiety and Related Disorders, 461-475.

Nelson, G. H., O’Hara, M. W., \& Watson, D. (2018). National norms for the Expanded Version of the Inventory of Depression and Anxiety Symptoms (IDAS-II). Journal of Clinical Psychology, 74, 953-968.

Ollendick, T. H., \& Hirshfeld-Becker, D. R. (2002). The developmental psychopathology of social anxiety disorder. Biological Psychiatry, 51(1), 44-58.

Otto, M. W., Pollack, M. H., Gould, R. A., Worthington III, J. J., McArdle, E. T., Rosenbaum, J. F., \& Heimberg, R. G. (2000). A comparison of the efficacy of clonazepam and cognitive-behavioral group therapy for the treatment of social phobia. Journal of Anxiety Disorders, 14(4), 345-358.

Porter, E., \& Chambless, D. L. (2017). Social anxiety and social support in romantic relationships. Behavior Therapy, 48(3), 335-348.

Richey, J. A., Brewer, J. A., Sullivan-Toole, H., Strege, M. V., Kim-Spoon, J., White, S. W., \& Ollendick, T. H. (2019). Sensitivity shift theory: A developmental model of positive affect and motivational deficits in social anxiety disorder. Clinical Psychology Review, 101756. 
Richey, J., Ghane, M., Valdespino, A., Coffman, M. C., Strege, M. V., White, S. W., \& Ollendick, T. H. (2017). Spatiotemporal dissociation of brain activity underlying threat and reward in social anxiety disorder. Social Cognitive and Affective Neuroscience, 12(1), 81-94.

Sleep, C. E., Lynam, D. R., Hyatt, C. S., \& Miller, J. D. (2017). Perils of partialing redux: The case of the Dark Triad. Journal of Abnormal Psychology, 126, 939-950.

Somerville, L. H. (2013). The teenage brain: Sensitivity to social evaluation. Current Directions in Psychological Science, 22(2), 121-127.

Springer, K. S., Levy, H. C., \& Tolin, D. F. (2018). Remission in CBT for adult anxiety disorders: A meta-analysis. Clinical Psychology Review, 61, 1-8.

Stanton, K. (2020). Increasing diagnostic emphasis on negative affective dysfunction: Potentially negative consequences for differential diagnosis. Clinical Psychological Science, 8, 548589.

Stanton, K., Stasik-O’Brien, S. M., Ellickson-Larew, S., \& Watson, D. (2016). Positive Affectivity: Specificity of its facet level relations with psychopathology. Cognitive Therapy and Research, 40(5), 593-605.

Stanton, K., \& Watson, D. (2015). Replicable facets of positive emotionality and their relations to psychopathology. Assessment, 22(6), 665-680.

Stasik-O’Brien, S. M., Brock, R. L., Chmielewski, M., Naragon-Gainey, K., Koffel, E., McDade-Montez, E., O’Hara, M. W., \& Watson, D. (2019). Clinical utility of the Inventory of Depression and Anxiety Symptoms (IDAS). Assessment, 26, 944-960. 
Strege, M. V., Swain, D., Bochicchio, L., Valdespino, A., \& Richey, J. A. (2018). A pilot study of the effects of mindfulness-based cognitive therapy on positive affect and social anxiety symptoms. Frontiers in Psychology, 9, 866.

Taylor, C. T., Knapp, S. E., Bomyea, J. A., Ramsawh, H. J., Paulus, M. P., \& Stein, M. B. (2017). What good are positive emotions for treatment? Trait positive emotionality predicts response to cognitive behavioral therapy for anxiety. Behaviour Research and Therapy, 93, 6-12.

Taylor, C. T., Pearlstein, S. L., \& Stein, M. B. (2017). The affective tie that binds: Examining the contribution of positive emotions and anxiety to relationship formation in social anxiety disorder. Journal of Anxiety Disorders, 49, 21-30.

Trew, J. L., \& Alden, L. E. (2012). Positive affect predicts avoidance goals in social interaction anxiety: Testing a hierarchical model of social goals. Cognitive Behaviour Therapy, 41(2), 174-183.

Tyrer, P., Nur, U., Crawford, M., Karlsen, S., MacLean, C., Rao, B., \& Johnson, T. (2005). The Social Functioning Questionnaire: a rapid and robust measure of perceived functioning. International Journal of Social Psychiatry, 51(3), 265-275.

Watson, D., Clark, L. A., \& Tellegen, A. (1988). Development and validation of brief measures of positive and negative affect: the PANAS scales. Journal of Personality and Social Psychology, 54(6), 1063.

Watson, D., \& Clark, L. A. (1999). The PANAS-X: Manual for the Positive and Negative Affect Schedule-Expanded Form. Retrieved from Iowa Research Online: http:/ /ir.uiowa.edu/psychology_pubs/11/ 
Watson, D., Clark, L. A., \& Stasik, S. M. (2011). Emotions and the emotional disorders: A quantitative hierarchical perspective. International Journal of Clinical and Health Psychology, 11(3), 429-442.

Watson, D., \& Naragon-Gainey, K. (2010). On the specificity of positive emotional dysfunction in psychopathology: Evidence from the mood and anxiety disorders and schizophrenia/schizotypy. Clinical Psychology Review, 30(7), 839-848.

Watson, D., \& Naragon-Gainey, K. (2014). Personality, emotions, and the emotional disorders. Clinical Psychological Science, 2(4), 422-442.

Watson, D., O’Hara, M. W., Naragon-Gainey, K., Koffel, E., Chmielewski, M., Kotov, R., ... Ruggero, C. J. (2012). Development and validation of new anxiety and bipolar symptom scales for an Expanded Version of the IDAS (the IDAS-II). Assessment, 19, 399-420.

Watson, D., O’Hara, M. W., Simms, L. J., Kotov, R., Chmielewski, M., McDade-Montez, E. A., Gamez, W., \& Stuart, S. (2007). Development and validation of the Inventory of Depression and Anxiety Symptoms (IDAS). Psychological Assessment, 19, 253-268. 

ANXIETY

\section{Tables}

Table 1. Correlations among the PA facets and NA.

\begin{tabular}{|c|c|c|c|c|c|}
\hline Factor & 1 & 2 & 3 & 4 & 5 \\
\hline 1. Joviality & - & $.78^{* *}$ & $.57 * *$ & $.63^{* *}$ & $-.30 * *$ \\
\hline 2. Self-Assurance & $.64 * *$ & - & $.61 * *$ & $.57 * *$ & $-.24 * *$ \\
\hline 3. Attentiveness & $.52 * *$ & $.58^{* *}$ & - & $.44^{* *}$ & $-.25^{* *}$ \\
\hline 4. Serenity & $.49 * *$ & $.45^{* *}$ & $.38^{* *}$ & - & $-.56 * *$ \\
\hline 5. Negative Affectivity & $-.14^{* *}$ & $-.15^{* *}$ & $-.11^{*}$ & $-.29 * *$ & - \\
\hline
\end{tabular}

$\overline{N=447 \text { and 375, respectively. All significant correlations }(\mathrm{p}<0.05) \text { are in bold. All significant correlations at the }(\mathrm{p}<}$ $.01)$ level are indicated in bold with one asterisk $(*)$; all significant correlations at the $(\mathrm{p}<.001)$ level are indicated in bold and with two asterisks(**). Results from the undergraduate sample are presented underneath the dashed lines, and results from the community sample are present on top of the dashed lines. 

ANXIETY

Table 2. Correlations for PA scales with self-rated psychopathology and social functioning.

\begin{tabular}{|c|c|c|c|c|c|}
\hline Measure & Joviality & Self-Assurance & Attentiveness & Serenity & Negative Affectivity \\
\hline \multicolumn{6}{|c|}{ Dimensional Symptom Scores } \\
\hline IDAS-II Social Anxiety & $-.26 * *(-.34) * *$ & $-.31 * *(-.34) * *$ & $-.22 * *(-.33) * *$ & $-.22 * *(-.47) * *$ & $.52 * *(.66) * *$ \\
\hline IDAS-II Dysphoria & $-.25 * *(-.40)^{* *}$ & $-.25 * *(-.31)^{* *}$ & $-.20 * *(-.34) * *$ & $-.34 * *(-.55)^{* *}$ & $.65^{* *}(.78)^{* *}$ \\
\hline \multicolumn{6}{|c|}{ Categorical Symptom Scores } \\
\hline Clinical Social Anxiety & $-.19 * *(-.21) * *$ & $-.21 * *(-.23) * *$ & $-.05(-.14) * *$ & $-.19 * *(-.27) * *$ & $.36 * *(.35) * *$ \\
\hline Clinical Dysphoria & $-.25 * *(-.21) * *$ & $-.20 * *(-.18) * *$ & $-.23 * *(-.15) * *$ & $-.34 * *(-.26) * *$ & $.61 *(.55) * *$ \\
\hline \multicolumn{6}{|c|}{ Social Functioning Scores } \\
\hline SFQ & $-.41 * *$ & $-.33 * *$ & $-.39 * *$ & $-.44 * *$ & $.53^{* *}$ \\
\hline DAS-17 Perfectionism & $-.26 * *$ & $-.16 * *$ & $-.20 * *$ & $-.27 * *$ & $.41 * *$ \\
\hline DAS-17 Dependency & $-.09 *$ & $-.14 * *$ & $-.11 *$ & $-.22 * *$ & $.33 * *$ \\
\hline
\end{tabular}

$N=447$ and 375 in the undergraduate and community samples, respectively. Correlation coefficients in parentheses are those from the community sample. All significant correlations $(p<0.05)$ are in bold. All significant correlations at the $(p<.01)$ level are indicated in bold with one asterisk $(*)$; all significant correlations at the $(\mathrm{p}<.001)$ level are indicated in bold and with two asterisks (**). IDAS-II Expanded Version of the Inventory of Depression and Anxiety Symptoms, SFQ Social Functioning Questionnaire, DAS-17 Dysfunctional Attitudes Scale. Note: Point biserial correlations were run for Social Anxiety Level and Dysphoria Level variables, which are dichotomous variables of the "clinical" cutoffs on the $I D A S-I I$. 
ANXIETY

Table 3. Standardized $\beta$ weights from multiple regression analyses.

\begin{tabular}{lllll}
\hline Measure & Joviality & Self-Assurance & Attentiveness & Serenity Negative Affectivity
\end{tabular}

\section{Dimensional Symptom Scores}

\begin{tabular}{|c|c|c|c|c|c|}
\hline IDAS-II Social Anxiety & $-.07(.03)$ & $-.20 * *(-.15)$ & $-.04(-.09)$ & $.07(-.03)$ & $.49 * *(.60)^{* *}$ \\
\hline IDAS-II Dysphoria & $-.08(-.15)^{*}$ & $-.05(.05)$ & $-.03(-.09)$ & $-.10(-.05)$ & $.60 * *(.70)^{* *}$ \\
\hline \multicolumn{6}{|c|}{ Social Functioning Scores } \\
\hline SFQ & $-.19 * *$ & .05 & $-.20 * *$ & $-.17 * *$ & $.44 * *$ \\
\hline DAS-17 Perfectionism & $-.17 *$ & .09 & -.08 & -.10 & $.36 * *$ \\
\hline DAS-17 Dependency & .08 & -.07 & -.02 & $-.14^{*}$ & $.28 * *$ \\
\hline
\end{tabular}

$\mathrm{N}=447$ and 375 in the undergraduate and community samples, respectively. Coefficients in parentheses are those from the community sample. Significant effects $(p<.05)$ are bolded. All significant effects at the $(p<.01)$ level are indicated in bold with one asterisk $\left(^{*}\right)$; all significant effects at the $(\mathrm{p}<.001)$ level are indicated in bold and with two asterisks (**). IDAS-II Expanded Version of the Inventory of Depression and Anxiety Symptoms, SFQ Social Functioning Questionnaire, $D A S$-17 Dysfunctional Attitudes Scale.

Table 4. Odds ratios from logistic regression analyses.

\begin{tabular}{|c|c|c|c|c|c|}
\hline Measure & Joviality & Self-Assurance & Attentiveness & Serenity & Negative Affectivity \\
\hline Social Anxiety & $.70(1.14)$ & $.59 *(.29)$ & $1.56(.98)$ & $1.00(.91)$ & $2.98 * *(2.62) * *$ \\
\hline Dysphoria & $.73(.58)$ & $1.00(.92)$ & $.98(.77)$ & $.81(.84)$ & $4.49 * *(6.94) * *$ \\
\hline
\end{tabular}

$N=447$ and 375 in the undergraduate and community samples, respectively. Coefficients in parentheses are those from the community sample. Significant effects $(p<0.05)$ are in bold. All significant effects at the $(\mathrm{p}<.01)$ level are indicated in bold with one asterisk $(*)$; all significant effects at the $(\mathrm{p}<.001)$ level are indicated in bold and with two $\operatorname{asterisks}(* *)$ 
Table 5. Contrast correlation analyses.

\begin{tabular}{lcc}
\hline \multicolumn{1}{c}{ Measure } & $\mathrm{Z}$ & P-value (One-tailed) \\
\hline Dimensional Symptom Scores & & $\mathbf{0 . 0 3}(\mathbf{0 . 0 5})$ \\
\hline IDAS-II Social Anxiety & $\mathbf{- 1 . 9 0}(\mathbf{1 . 7 0})$ & $0.28(\mathbf{0 . 0 1})$ \\
\hline IDAS-II Dysphoria & $0.58(\mathbf{2 . 5 1})$ & $\mathbf{0 . 0 1}(0.33)$ \\
\hline Categorical Symptom Scores & & $\mathbf{0 . 0 4}(0.39)$ \\
\hline Clinical Social Anxiety & $\mathbf{- 2 . 3 7}(-0.44)$ & \\
\hline Clinical Dysphoria & $\mathbf{1 . 7 5}(0.30)$ & 0.73 \\
\hline Social Functioning Scores & 1.12 & 0.23 \\
\hline SFQ & 0.73 & 0.07 \\
\hline DAS-17 Perfectionism & 1.48 & \\
\hline DAS-17 Dependency & & \\
\hline
\end{tabular}

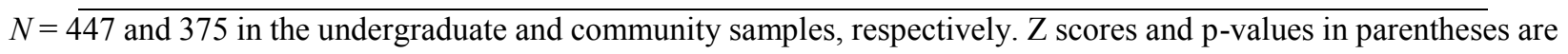
those from the community sample, whereas those not within parentheses are from the undergraduate sample. We computed Z- and p-values (one-tailed test) to test whether the correlation between motivationally-valenced PA facets (i.e., self-assurance and joviality) and respective measures differed significantly from the correlation between the nonmotivationally-valenced (i.e., attentiveness and serenity) and respective measures. We used the formula to test the significance of difference between dependent r's (Meng, Rosenthal, \& Rubin, 1992). Significant effects $(p<0.05)$ are in bold. IDAS-II Expanded Version of the Inventory of Depression and Anxiety Symptoms, SFQ Social Functioning Questionnaire, DAS-17 Dysfunctional Attitudes Scale. Note: Point biserial correlations were run for Social Anxiety Level and Dysphoria Level variables, which are dichotomous variables of the "clinical" cutoffs on the IDAS-II. 